\title{
Critical Temperature of Dirty Bosons
}

\author{
B. KLÜNDER ${ }^{1}$, A. PELSTER ${ }^{2}$, and R. GRAHAM ${ }^{3}$ \\ Fachbereich Physik, Universität Duisburg-Essen, Campus Duisburg \\ Lotharstraße 1, 47048 Duisburg, Germany \\ ${ }^{1}$ E-mail: kluender@theo.phys.uni-duisburg-essen.de \\ ${ }^{2}$ E-mail: axel.pelster@uni-duisburg-essen.de \\ ${ }^{3}$ E-mail: robert.graham@uni-duisburg-essen.de
}

\begin{abstract}
We consider a dilute Bose Gas moving in a harmonic trap with a superimposed frozen random potential which arises in experiments either naturally in wire traps or artificially and controllably with laser speckles. The critical temperature, which characterises the onset of Bose-Einstein condensation, depends on the disorder realization within the ensemble. Therefore, we introduce an effective grand-canonical potential from which we determine perturbatively the disorder averages of both the first and the second moment of the critical temperature in leading order. We discuss our results for a finite number of particles by assuming a Gaussian spatial correlation for the quenched disorder potential.

Keywords: Disorder, Bose-Einstein condensation, critical temperature
\end{abstract}

\section{Introduction}

This work is motivated by recent experiments which study disorder effects on Bose-Einstein condensates (BECs). Frozen random potentials for BECs arise naturally in wire traps due to surface roughness. ${ }^{1,2}$ For a better understanding of these effects one needs to create disorder in a more controllable fashion. A prominent example is provided by laser speckles where both strength and correlation length of disorder realizations can be tuned. ${ }^{3,4}$

Here we investigate the effect of disorder on the critical temperature of a harmonically trapped dilute ideal Bose gas. We assume that the disorder potential is spatially Gaussian correlated and that it is weak enough to treat it in a perturbative manner. The aim of this work is to determine the disorder-induced shift of the critical temperature and the mean deviation around its average. To this end we calculate the critical temperature as a functional of the disorder potential and evaluate then the first and second disorder averaged moments. 


\section{Effective Action}

We start with the usual functional integral representation of the grandcanonical partion function

$$
Z=\oint \mathcal{D} \psi^{*} \oint \mathcal{D} \psi e^{-\mathcal{A} / \hbar}
$$

where one integrates over all possible bosonic Schrödinger fields which are periodic in imaginary time. The corresponding action reads

$\mathcal{A}=\int_{0}^{\hbar \beta} d \tau \int d^{3} x \psi^{*}(\mathbf{x}, \tau)\left[\hbar \frac{\partial}{\partial \tau}-\frac{\hbar^{2}}{2 M} \Delta+\frac{M}{2} \omega^{2} \mathbf{x}^{2}+U(\mathbf{x})-\mu\right] \psi(\mathbf{x}, \tau)$

where $U(\mathbf{x})$ and $\omega$ denote the disorder potential and the frequency of the isotropic harmonic trap.

To find the average influence of the disorder we consider a large ensemble of macroscopically identical realizations and define an ensemble average as

$$
\longrightarrow=\prod_{\mathbf{x}} \int_{\infty}^{\infty} d U(\mathbf{x}) \bullet P[U], \quad \overline{1}=1
$$

For our purposes it is not necessary to know the concrete form of the probability distribution $P[U]$ apart from the condition that it has to be bounded from below. Furthermore, we assume that the disorder potential vanishes on the average and that the disorder is spatially Gaussian correlated: ${ }^{7}$

$$
\overline{U\left(\mathbf{x}_{1}\right)}=0, \quad \overline{U\left(\mathbf{x}_{1}\right) U\left(\mathbf{x}_{2}\right)}=\frac{R}{\left(2 \pi \epsilon^{2}\right)^{\frac{3}{2}}} e^{-\frac{\left(\mathbf{x}_{1}-\mathbf{x}_{2}\right)^{2}}{2 \epsilon^{2}}} .
$$

Here $R$ is the disorder strength and $\epsilon$ the disorder correlation length. In the next step we calculate the effective action and make use of the so-called background method. ${ }^{5,6}$ Thus, we use the decomposition $\psi(\mathbf{x}, \tau)=\Psi(\mathbf{x}, \tau)+\delta \psi(\mathbf{x}, \tau)$ for the Schrödinger field, where $\Psi(\mathbf{x}, \tau)$ and $\delta \psi(\mathbf{x}, \tau)$ denote field expectation value and fluctuations, respectively. The remaining functional integral in the partion function is expanded for a small disorder potential $U(\mathbf{x})$ up to second order. After applying the logarithm to the grand-canonical partion function we get for the effective action 


$$
\begin{aligned}
& \Gamma\left[\Psi^{*}, \Psi\right]=-\sum_{k=1}^{\infty} \frac{e^{\beta\left(\mu-E_{0}\right) k}}{\beta k}\left[\frac{1}{\left(1-e^{-\hbar \omega \beta k}\right)^{3}}-1\right] \\
& +\frac{1}{\hbar \beta} \int_{0}^{\hbar \beta} d \tau \int d^{3} x \Psi^{*}(\mathbf{x}, \tau)\left[\hbar \frac{\partial}{\partial \tau}-\frac{\hbar^{2}}{2 M} \Delta+\frac{M}{2} \omega^{2} \mathbf{x}^{2}+U(\mathbf{x})-\mu\right] \Psi(\mathbf{x}, \tau) \\
& -\frac{1}{2 \hbar^{2} \beta} \int_{0}^{\hbar \beta} d \tau_{1,2} \int d^{3} x_{1,2} U\left(\mathbf{x}_{1}\right) U\left(\mathbf{x}_{2}\right) G_{0}\left(\mathbf{x}_{1}, \tau_{1} ; \mathbf{x}_{2}, \tau_{2}\right) G_{0}\left(\mathbf{x}_{2}, \tau_{2} ; \mathbf{x}_{1}, \tau_{1}\right) \\
& +\int d^{3} x U(\mathbf{x}) G_{0}(\mathbf{x}, 0 ; \mathbf{x}, 0)+\ldots
\end{aligned}
$$

Here $G_{0}\left(\mathbf{x}_{1}, \tau_{1} ; \mathbf{x}_{2}, \tau_{2}\right)=\left\langle\psi\left(\mathbf{x}_{1}, \tau_{1}\right) \psi^{*}\left(\mathbf{x}_{2}, \tau_{2}\right)\right\rangle$ stands for the unperturbed correlation function of the Schrödinger fields. The grand-canonical potential $\Omega$ can be found by extremizing the effective action (5) with respect to the field expectation values:

$$
\frac{\delta \Gamma\left[\Psi_{e}^{*}, \Psi_{e}\right]}{\delta \Psi_{e}(\mathbf{x}, \tau)}=\frac{\delta \Gamma\left[\Psi_{e}^{*}, \Psi_{e}\right]}{\delta \Psi_{e}^{*}(\mathbf{x}, \tau)}=0 \quad \Rightarrow \quad \Omega(T, V, \mu)=\Gamma\left[\Psi_{e}^{*}, \Psi_{e}\right] .
$$

In the symmetry-broken phase we expand the critical chemical potential $\mu_{c}$ and the extremal field expectation value $\Psi_{e}(\mathbf{x}, \tau)$ with respect to $U(\mathbf{x})$ up to second order to determine the respective disorder-induced corrections:

$\mu_{c}=\frac{3}{2} \hbar \omega+\mu_{c}^{(1)}+\mu_{c}^{(2)}+\ldots, \Psi_{e}(\mathbf{x}, \tau)=\sqrt{N_{0}} \psi_{0}(\mathbf{x})+\Psi_{e}^{(1)}(\mathbf{x})+\Psi_{e}^{(2)}(\mathbf{x})+\ldots$

Here $\psi_{0}(\mathbf{x})$ denotes the ground-state wave function and $3 \hbar \omega / 2$ the groundstate energy of the three-dimensional harmonic oscillator. Both corrections allow us in the following to calculate the critical temperature as a functional of the disorder potential.

\section{Critical Temperature}

We determine the critical temperature from the particle number equation $N$ which follows immediately from the grand-canonical potential $\Omega$. To this end we set the ground-state occupation number $N_{0}$ equal to zero, the chemical potential equal to its critical value $\mu_{c}$, and expand the critical temperature: $T_{c}[U]=T_{c}^{(0)}+T_{c}^{(1)}[U]+T_{c}^{(2)}[U]+\ldots$ with $T_{c}^{(0)}=\hbar \omega N^{1 / 3} / \zeta(3)^{1 / 3} k_{B}$. Using the disorder averages (4) one finds the following equations for the average shift and the variance:

$$
\frac{\overline{\Delta T_{c}[U]}}{T_{c}^{(0)}}=\frac{\overline{T_{c}^{(2)}[U]}}{T_{c}^{(0)}}+\ldots, \quad \frac{\overline{\Delta T_{c}[U]^{2}}}{T_{c}^{(0) 2}}=\frac{\overline{T_{c}^{(1)}[U]^{2}}}{T_{c}^{(0) 2}}+\ldots
$$


a)

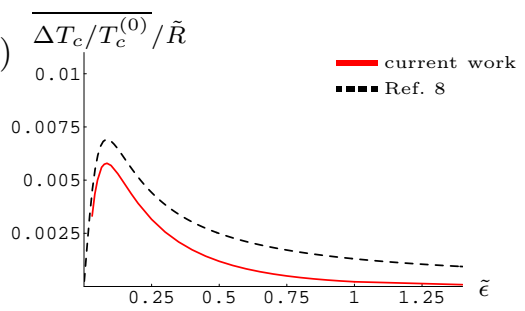

b) $\sqrt{\overline{\left(\Delta T_{c} / T_{c}^{(0)}\right)^{2}} / \tilde{R}}$

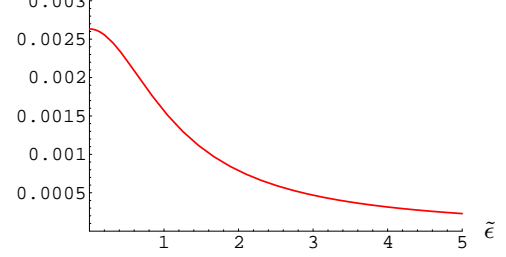

Fig. 1. Average shift of critical temperature a) and mean deviation b) versus $\tilde{\epsilon}=\epsilon \sqrt{M \omega / \hbar}$ for $N=10^{5}$ and $\tilde{R}=R M^{3 / 2} \omega^{-1 / 2} \hbar^{-7 / 2}$.

The respective results can be seen in Fig. 1. The average shift of the critical temperature in Fig. 1 a) is positive and has a maximum when the correlation length $\epsilon$ is about the same size than the thermal wave length $\lambda_{c}^{(0)}$ of the bosons at the critical temperature. Both the critical temperature and the mean deviation are proportional to $N^{-1 / 3}$ when $\tilde{\epsilon}$ is kept constant. In the related Ref. $^{8}$ it is assumed that the critical temperature is self-averaging. This approximation leads qualitatively to the same result but differs quantitatively as is shown in Fig. 1 a) for an experimentally realistic particle number. One also finds in Fig. 1 b) that the relative variance does not vanish for disorder correlation lengths much smaller than the oscillator length. This implies that the critical temperature is not a self-averaging quantity with respect to the correlation length.

\section{Acknowledgements}

This work was supported by the SFB/TR 12 of the German Research Foundation (DFG) and by the Wilhelm and Else Heraeus Foundation.

\section{References}

1. P. Kruger et al., cond-mat/0504686

2. J. Fortagh, H. Ott, S. Kraft, and C. Zimmermann, Phys. Rev. A 66, R041604 (2002)

3. J. E. Lye et al., Phys. Rev. Lett. 95, 070401 (2005)

4. D. Clément et al., Phys. Rev. Lett. 95, 170409 (2005)

5. C. Morette, Phys. Rev. 81, 848 (1951)

6. B. S. DeWitt, Theory of Dynamical Groups and Fields (Gordon and Breach, New York, 1965)

7. M. Kobayashi and M. Tsubota, Phys. Rev. B 66, 174516 (2002)

8. M. Timmer, A. Pelster, and R. Graham, Europhys. Lett. 76, 760 (2006) 